$12-2003$

\title{
Simulated Properties of Kagomé and Tetragonal Truss Core Panels
}

S. Hyun

Johns Hopkins University

Anette M. Karlsson

Cleveland State University, a.karlsson@csuohio.edu

S. Torquato

Princeton University

A. G. Evans

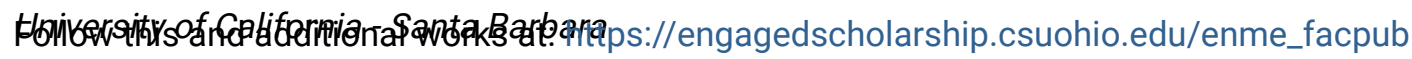

Part of the Mechanical Engineering Commons

How does access to this work benefit you? Let us know!

Publisher's Statement

NOTICE: this is the author's version of a work that was accepted for publication in International Journal of Solids and Structures. Changes resulting from the publishing process, such as peer review, editing, corrections, structural formatting, and other quality control mechanisms may not be reflected in this document. Changes may have been made to this work since it was submitted for publication. A definitive version was subsequently published in International Journal of Solids and Structures, 40, 25, (12-01-2003); 10.1016/S0020-7683(03)00350-0

\section{Original Citation}

Hyun, S., Karlsson, A. M., Torquato, S., 2003, "Simulated Properties of Kagomé and Tetragonal Truss Core Panels," International Journal of Solids and Structures, 40(25) pp. 6989-6998.

This Article is brought to you for free and open access by the Mechanical Engineering Department at EngagedScholarship@CSU. It has been accepted for inclusion in Mechanical Engineering Faculty Publications by an authorized administrator of EngagedScholarship@CSU. For more information, please contact library.es@csuohio.edu. 


\title{
Simulated properties of Kagomé and tetragonal truss core panels
}

\author{
S. Hyun ${ }^{\text {a }}$, A.M. Karlsson ${ }^{\text {b }}$, S. Torquato ${ }^{\text {c,* }}$, A.G. Evans ${ }^{\text {d }}$ \\ a Department of Physics \& Astronomy, Johns Hopkins University, Baltimore, MD 21218, USA \\ b Department of Mechanical Engineering, University of Delaware, Newark, DE 19716-3140, USA \\ ' Department of Chemistry and Princeton Materials Institute, Princeton University, Princeton, New Jersey, USA \\ ${ }^{d}$ Materials Department, University of California at Santa Barbara, Santa Barbara, CA 93106, USA
}

\section{Introduction}

Progress in robust, ultra-light metallic materials and systems with topologically configured cores and dense faces has provided several perspectives (Wicks and Hutchinson, 2000; Wallach and Gibson, 2001; Deshpande et al., 2001; Evans et al., 2001; Deshpande and Fleck, 2001; Chiras et al., 2002). Panels having cores with a stochastic cellular topology are not weight efficient, but have utility because of relatively low cost and excellent robustness (Ashby et al., 2000). Periodic truss core panels having tetragonal and pyramidal topology exhibit superior thermo-structural characteristics (Deshpande and Fleck, 2001; Chiras et al., 2002). They are at least as weight efficient as the best competing concepts, especially for curved panels (Evans et al., 1998).

The preferred core topologies are based on the fundamental idea that the trusses should stretch/compress without bending. When realized, the core properties are related to its relative density, $\bar{\rho}_{\text {core }}$, by Wicks and

\footnotetext{
"Corresponding author. Tel.: +1-609-258-3341; fax: +1-609-258-6878.

E-mail address: torquato@matter.princeton.edu (S. Torquato).
} 


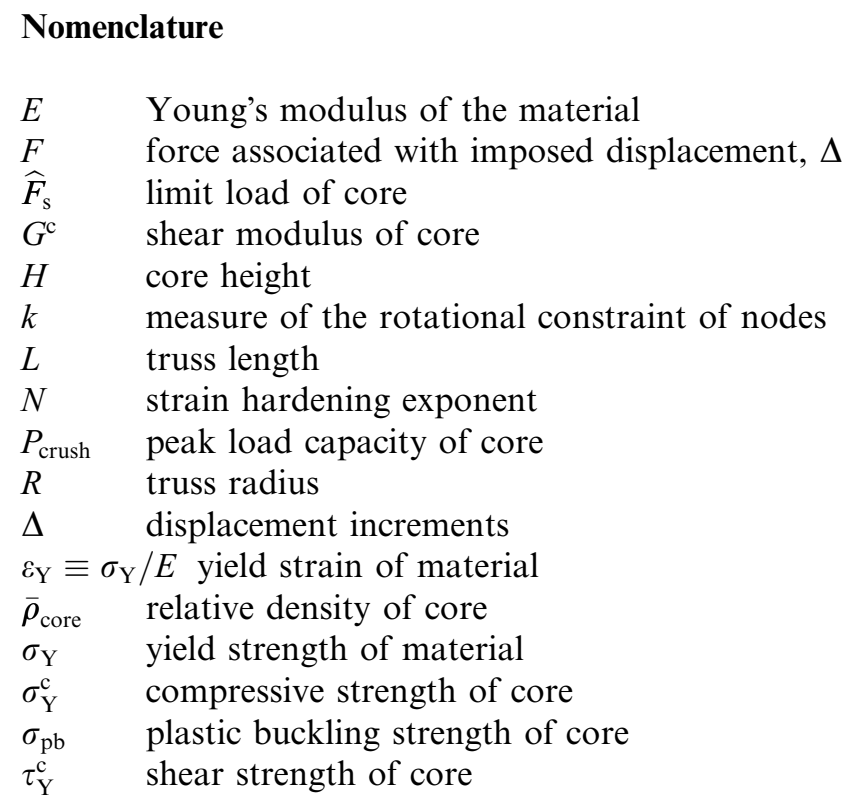

Hutchinson (2000), Wallach and Gibson (2001), Deshpande et al. (2001), Evans et al. (2001), Deshpande and Fleck (2001), Chiras et al. (2002) and Evans et al. (1998):

$$
\begin{aligned}
& G^{\mathrm{c}} / E=A \bar{\rho}_{\text {core }}, \\
& \tau_{\mathrm{Y}}^{\mathrm{c}} / \sigma_{\mathrm{Y}}=B \bar{\rho}_{\text {core }}, \\
& \sigma_{\mathrm{Y}}^{\mathrm{c}} / \sigma_{\mathrm{Y}}=C \bar{\rho}_{\text {core }},
\end{aligned}
$$

where $\sigma_{\mathrm{Y}}$ is the yield strength and $E$ the Young's modulus of the material comprising the trusses, with $G^{\mathrm{c}}$ the shear modulus, $\tau_{\mathrm{Y}}^{\mathrm{c}}$ the shear strength and $\sigma_{\mathrm{Y}}^{\mathrm{c}}$ the compressive strength. The coefficients $A, B$ and $C$ are functions of truss architecture, loading orientation and node design. Adding the constraint that the core should be nearly isotropic (to minimize compliant orientations), only a small subset of possible truss core topologies appears to satisfy Eqs. (1) (Evans et al., 1998; Evans, 2001). Two have been analyzed and characterized experimentally: tetragonal (Deshpande and Fleck, 2001; Chiras et al., 2002) (Fig. 1a) and pyramidal (Deshpande and Fleck, 2001). The shear and compressive response of these cores has been determined, as well as the bending characteristics of panels in near-optimized configurations. The performance of panels with these cores is excellent (Chiras et al., 2002). Nevertheless, improvements appear feasible, based on the following two limitations.

(i) The cores have significant anisotropy. The coefficients $A$ and $B$ in (1) vary with loading orientation: for the pyramidal design differing by as much as $40 \%$ between the maximum and minimum.

(ii) The trusses are susceptible to plastic buckling, resulting in a bending asymmetry, particularly for the tetragonal topology.

In order to address these limitations, an objective of this study is to explore the comparative performance of an alternative core topology, known as the 3D Kagomé (Fig. 1b). The genesis of this choice has 


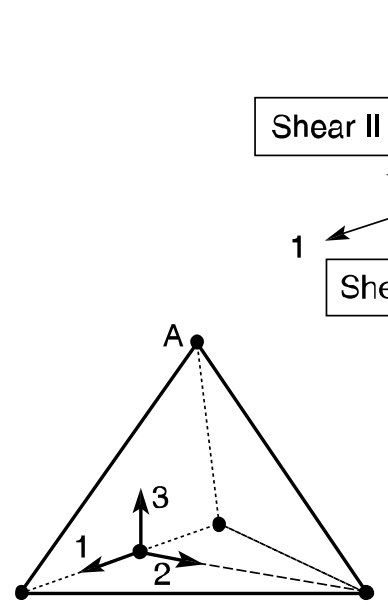

(a)

\section{Shear I}

2

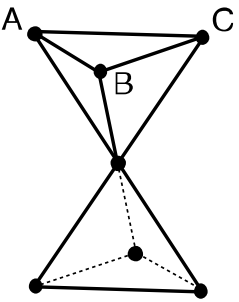

(b)

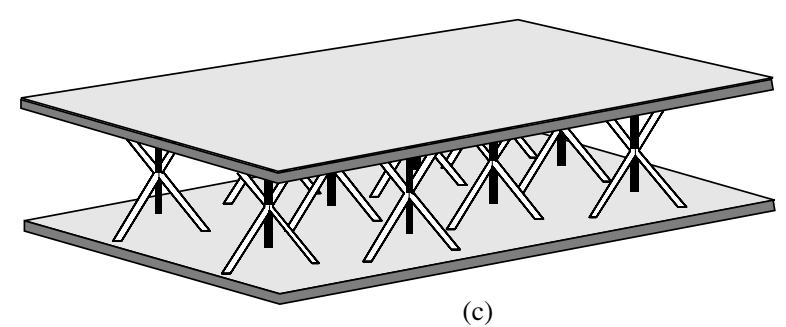

(c)

Fig. 1. Illustrations of the tetragonal (a) and Kagomé topologies (b), showing the shear load orientations (I, II, III) applied on the nodes (A for the tetragonal core and A, B, C for the Kagomé core); (c) illustrates the Kagomé truss core panel.

been the recent finding from topology optimization that 2D Kagomé structures are structurally efficient (Hyun and Torquato, 2002). Namely, their elastic moduli approach the optimal Hashin-Shtrikman upper bounds (Hashin and Shtrikman, 1963; Hashin, 1965) over a wide range of relative densities and they have superior buckling properties. In order to fully capture the failure mechanisms, detailed finite element models, using solid elements, of the two structures (tetragonal and Kagomé) are developed. The models are confirmed by means of experimental measurements described in a companion paper (Wang et al., 2003).

\section{Problem definition}

The dimensions of the tetragonal core (core radius, rod length, panel height) are representative of nearoptimized sandwich panels (Wicks and Hutchinson, 2000), with relative density, $\rho_{\text {core }} \approx 0.02$. The same truss radius and panel height are used for the Kagomé core, but to attain the same core density, the truss length is half that for the tetragonal core (Hyun and Torquato, 2002). The displacements imposed on the model are chosen to simulate core compression and shear. For compression, a vertical displacement is applied to the top plane. This plane is prohibited from transverse motion and rotation, while the bottom remains rigid. The shear displacements are applied in three principal directions, reflecting the extrema. These are positive (orientation I) and negative (orientation II) along direction 2 and positive (orientation III) along direction 1 (see Fig. 1). The boundary conditions reflect the constraint exerted by the faces. That is, the rotation of the plane representing the face sheet is prohibited, consistent with a face sheet that remains planar. The bottom plane remains rigid. 


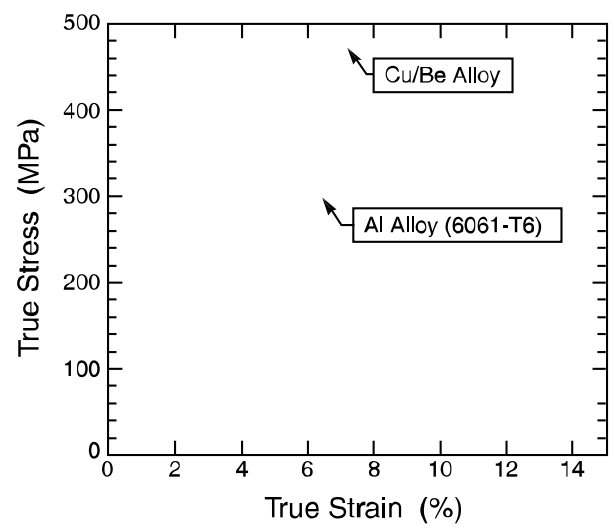

Fig. 2. Stress/strain curves for $\mathrm{Cu} / \mathrm{Be}$ alloy and Al-alloy (6061-6T).

The stress/strain relations used in the simulations (Fig. 2) are representative of those for a $\mathrm{Cu} / \mathrm{Be}$ casting alloy with appreciable strain hardening (Chiras et al., 2002) and a high performance Al alloy (6061-6T) with much lower strain hardening. The stress/strain $[\sigma(\varepsilon)]$ curves are fit to a Ramberg-Osgood representation:

$$
\varepsilon=\sigma / E+\left(\sigma_{\mathrm{Y}} / E\right)\left(\sigma / \sigma_{\mathrm{Y}}\right)^{N},
$$

with the strain hardening exponent $N$. For $\mathrm{Cu} / \mathrm{Be}$, the fit to the stress/strain measurements (Chiras et al., 2002) indicates that: $E=130 \mathrm{GPa}, N=7.4, \sigma_{\mathrm{Y}}=290 \mathrm{MPa}$ and $\varepsilon_{\mathrm{Y}} \equiv \sigma_{\mathrm{Y}} / E=2.2 \times 10^{-3}$. The corresponding results for the Al-alloy are: $E=69 \mathrm{GPa}, N=28, \sigma_{\mathrm{Y}}=275 \mathrm{MPa}$ and $\varepsilon_{\mathrm{Y}} \equiv \sigma_{\mathrm{Y}} / E=3.98 \times 10^{-3}$.

\section{Simulation method}

The commercial package, ANSYS, was used to generate three-dimensional meshes by utilizing 10-node tetrahedral solid elements, with about 10,000 finite elements and 10,000 nodes needed to obtain reliable convergence. The meshed models were transferred to the commercial finite element solver, ABAQUS, to perform the numerical simulations. The simulations were performed subject to displacement-control, using large displacement theory to capture the softening in the post-buckling state. Small displacement increments, $\Delta$, were selected, especially near yield, to avoid numerical instability and the occurrence of local minima ( $\Delta / H$ ranged from 0.003 to 0.025 , where $H$ is the core height). The boundary conditions described in Section 2 were imposed. The associated force, $F$, was obtained from the sum of the forces on each node intersecting the face. Unload/reload simulations were performed for compressive loading, as well as for shear loading in orientation I. These numerical unload/reload tests reveal not only the Young's modulus of the system, but are used as a convergence check of the simulations.

\section{Results}

\subsection{Compression}

The calculated relations between the non-dimensional compressive force, $F / \pi \sigma_{\mathrm{Y}} R^{2}$ (where $R$ is the truss radius) and vertical displacement, $\Delta / H$ (Fig. 3) reveal that, for both truss designs, a maximum load capacity 


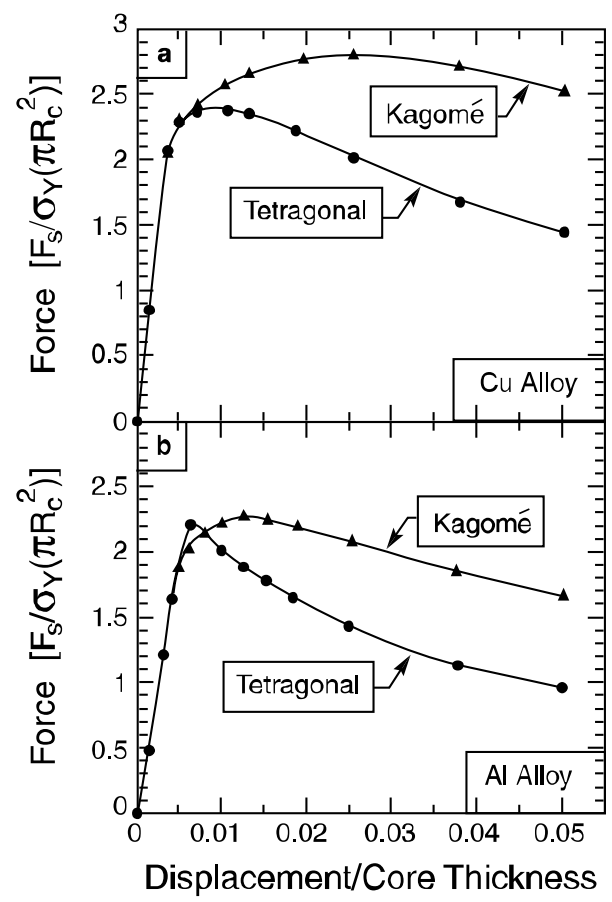

Fig. 3. Non-dimensional force/strain curves calculated in compression for tetragonal and Kagomé cores made from (a) Cu alloy and (b) $\mathrm{Al}$ alloy.

is reached, followed by softening. The peak loads are systematically lower for the $\mathrm{Al}$ alloy than the $\mathrm{Cu} / \mathrm{Be}$ alloy, and occur at lower $\Delta$, because of the differences in strain hardening.

The Kagomé geometry sustains appreciably higher loads than the tetrahedron, as well as exhibiting larger load carrying capacity before softening. The deformations exhibited by the trusses beyond the load maximum are illustrated on Fig. 4. Such deformations reveal that the peak loads coincide with the onset of

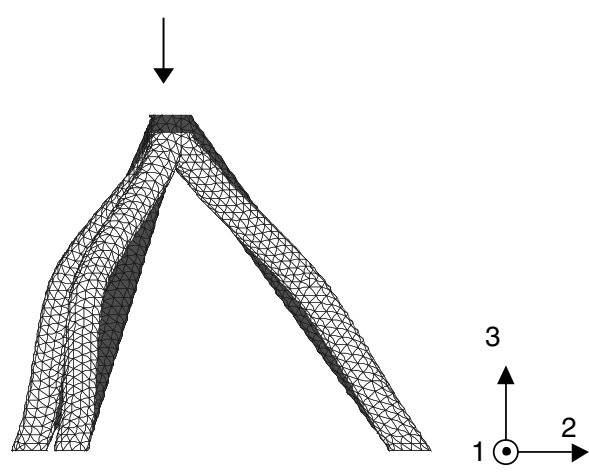

(a)

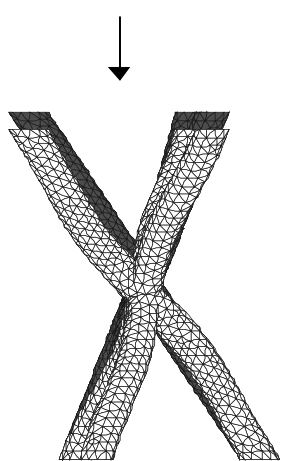

(b)

Fig. 4. The tetragonal (a) and Kagomé (b) configurations made from $\mathrm{Cu}$ alloys under compression at an imposed strain, $\Delta / H=0.05$, beyond the load maximum. Note that, for both cores, the trusses experience plastic buckling. 
Table 1

Plastic buckling strengths $\left(\sigma_{\mathrm{pb}}\right)$ for the truss members $(\mathrm{MPa})$

\begin{tabular}{llllll}
\hline$k$ & Cu/Be alloy & & & Al alloy \\
\cline { 2 - 3 } & Tetragonal & & Kagomé & & Ketragonal \\
\hline 1 & 225 & 287 & 230 & 255 \\
2 & 257 & 317 & 245 & 263 \\
3 & 275 & 336 & 251 & 267 \\
4 & 287 & 350 & 255 & 269 \\
\hline
\end{tabular}

plastic buckling. Accordingly, the peak loads might be rationalized in terms of the plastic buckling strength for the truss members, $\sigma_{\mathrm{pb}}$ (Deshpande and Fleck, 2001; Chiras et al., 2002). This strength relates to the properties of the truss material through the implicit expression:

$$
k\left(\frac{\pi R}{2 L}\right)^{2} \varepsilon_{\mathrm{Y}}^{-1}=\left(\frac{\sigma_{\mathrm{pb}}}{\sigma_{\mathrm{Y}}}\right)+N\left(\frac{\sigma_{\mathrm{pb}}}{\sigma_{\mathrm{Y}}}\right)^{N},
$$

where $L$ is the truss length, with $k$ a measure of the rotational constraint at the junctions $(k=1$ when there is no constraint and $k=4$ when clamped). It differs for the tetragonal and Kagomé geometries because of the different truss lengths: the aspect ratio $L / R$ is smaller in the Kagomé than the tetragonal structure, although $R$ is the same. The bounds on $\sigma_{\mathrm{pb}}$, with and without constraints, determined for both structures and both alloys are summarized in Table 1 .

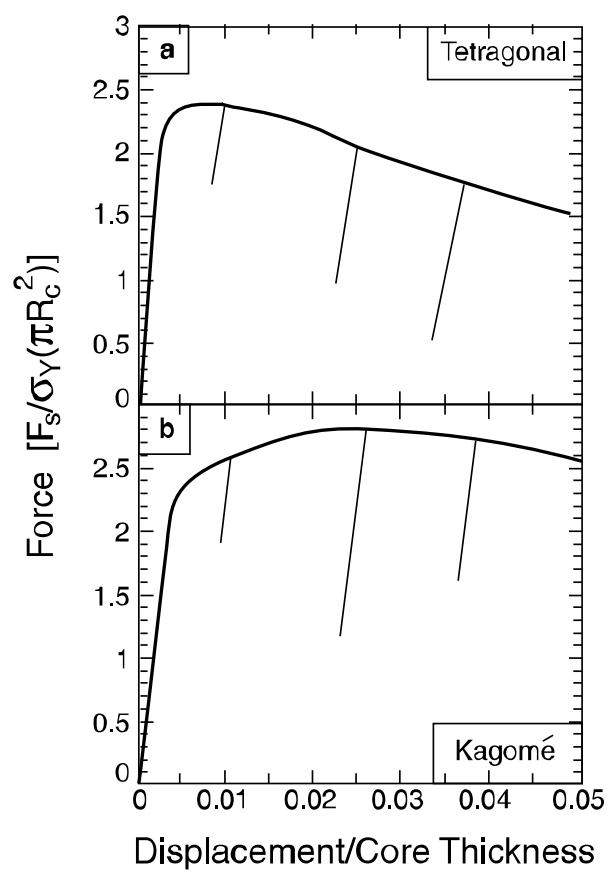

Fig. 5. Unload/reload simulations (Cu alloy) revealing that, for the tetragonal core (a), the unloading modulus decreases at strains beyond the onset of plastic buckling. For the Kagomé core (b), the modulus maintains virtually constant through the same displacements. 
The compressive strength $\sigma_{\mathrm{Y}}^{\mathrm{c}}$ of the optimized sandwich panel is given by (Wicks and Hutchinson, 2000):

$$
\sigma_{\mathrm{Y}}^{\mathrm{c}} / \sigma_{\mathrm{Y}}^{\mathrm{core}}=\frac{H^{2}}{2} \rho_{\text {core }} \text {. }
$$

The maximum stress needed to crush the core is obtained by using the stress for plastic buckling $\sigma_{\mathrm{pb}}$ in Eq. (3), determined for the clamped conditions $k=4$, and inserting into the result for the compressive strength (4). This predicts the peak stresses $P_{\text {crush }}=3.8 \mathrm{MPa}$ for the tetragonal core and $P_{\text {crush }}=4.7 \mathrm{MPa}$ for the Kagomé core (relative density $\rho_{\text {core }} \approx 0.02$ ). In the simulation, the maximum stresses needed to crush the tetragonal core and Kagomé core are given by $P_{\text {crush }}=4.3 \mathrm{MPa}$ and $P_{\text {crush }}=5.1 \mathrm{MPa}$. The larger value found in the simulations has yet to be explained.

The unload/reload simulations (Fig. 5) on $\mathrm{Cu} / \mathrm{Be}$ alloy reveal that the unloading modulus for the tetragonal core decreases once softening commences (Fig. 5a), but remains unchanged for the Kagomé core (Fig. 5b).

\subsection{Shear}

The non-dimensional stress/strain curves for shear loaded structures are summarized in Fig. 6. For the $\mathrm{Cu} / \mathrm{Be}$ alloy, the stress/strain curves are obtained for the three shear orientations. For the Al alloy, they are

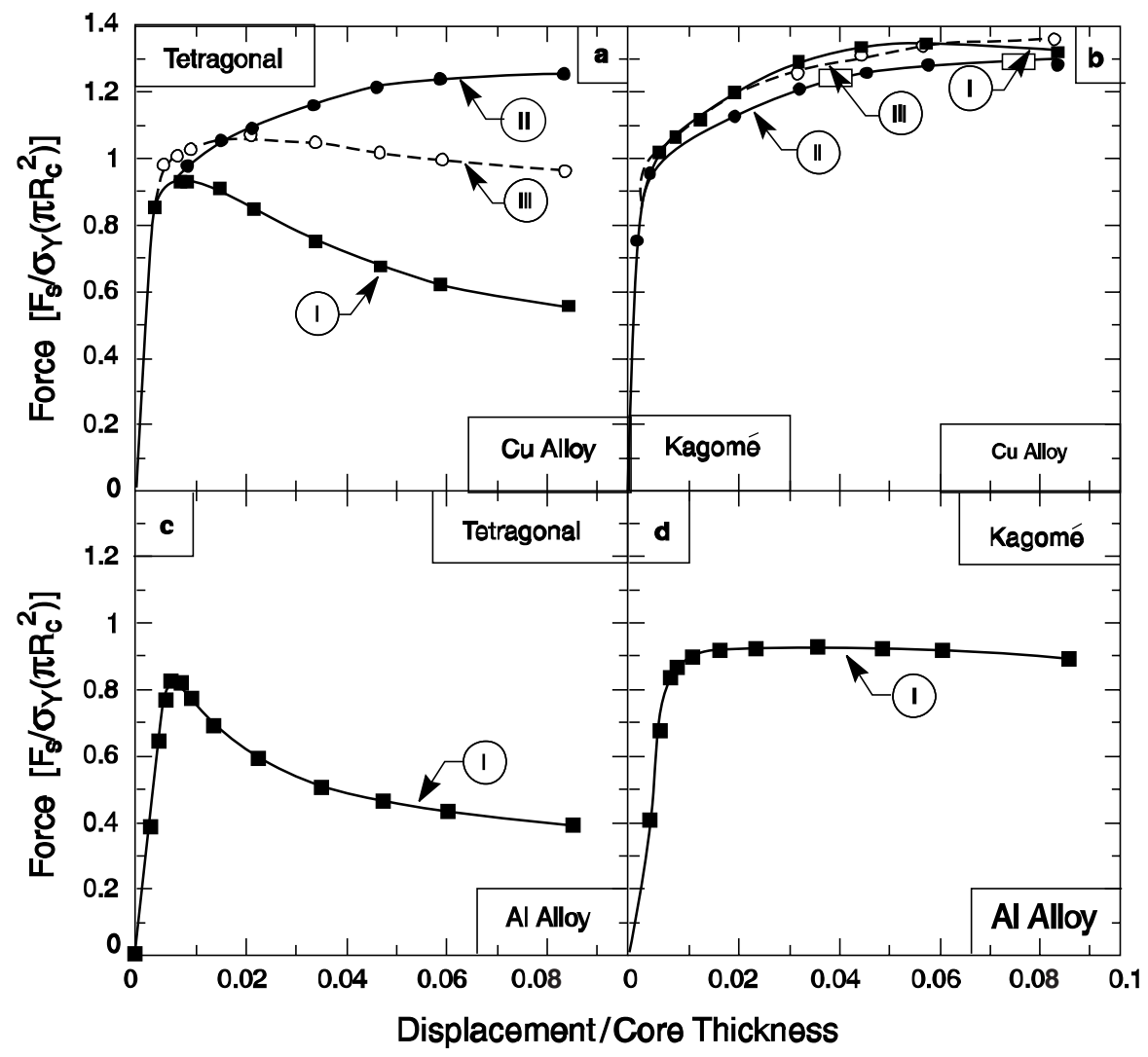

Fig. 6. Non-dimensional force/strain curves calculated in three shear orientations for the tetragonal and Kagomé cores made from $\mathrm{Cu}(\mathrm{a}, \mathrm{b})$ and $\mathrm{Al}(\mathrm{c}, \mathrm{d})$ alloys. 
obtained in the most compliant orientation I. Appreciable differences in response are evident between the tetragonal and Kagomé cores. The Kagomé topology is essentially isotropic, exhibiting similar response in all three orientations (Fig. 6b). For the $\mathrm{Cu} / \mathrm{Be}$ alloy, hardening occurs and a limit load, $\widehat{F}_{\mathrm{s}} / \pi \sigma_{\mathrm{Y}} R^{2} \approx 1.3$, is approached, without significant softening, at strains up to $10 \%$. These trends have been confirmed experimentally (Wang et al., 2003). For the Al alloy, the hardening is reduced and the load has a maximum, $\widehat{F}_{\mathrm{s}} / \pi \sigma_{\mathrm{Y}} R^{2} \approx 1.0$. The distortions of the trusses are similar in all orientations, with minimal deviation from direct shear.

The tetragonal topology is anisotropic and asymmetric (Fig. 6a). In orientations I and III, the load has a peak, followed by softening. The maximum loads in orientation I are, $\widehat{F}_{\mathrm{s}} / \pi \sigma_{\mathrm{Y}} R^{2} \approx 0.93$ for the $\mathrm{Cu} / \mathrm{Be}$ alloy (Fig. 6a) and $\widehat{F}_{\mathrm{s}} / \pi \sigma_{\mathrm{Y}} R^{2} \approx 0.8$ for the $\mathrm{Al}$ alloy (Fig. 6c). In orientation III, the maximum load is

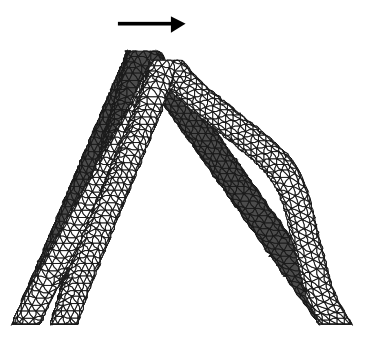

(a)

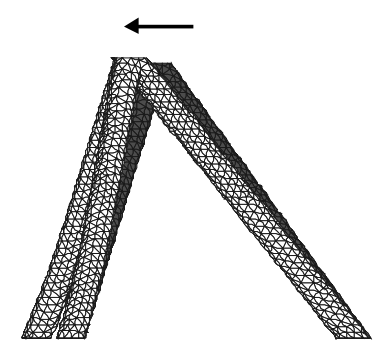

(c)

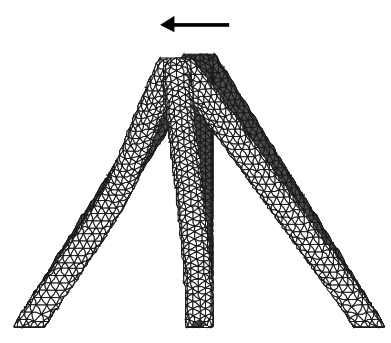

(e)

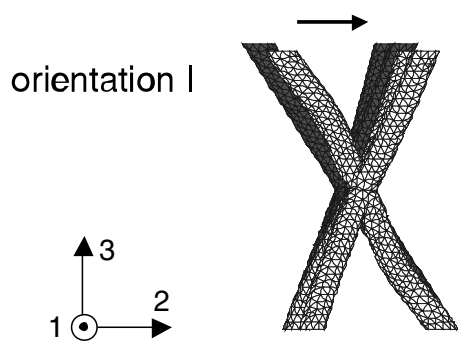

(b)

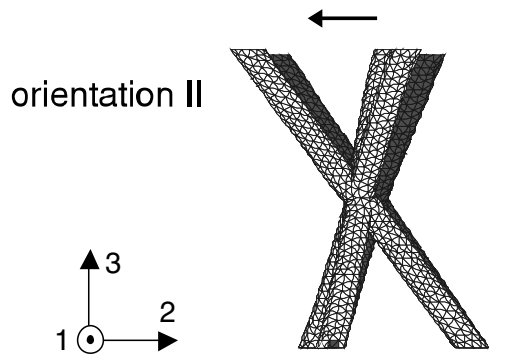

(d)
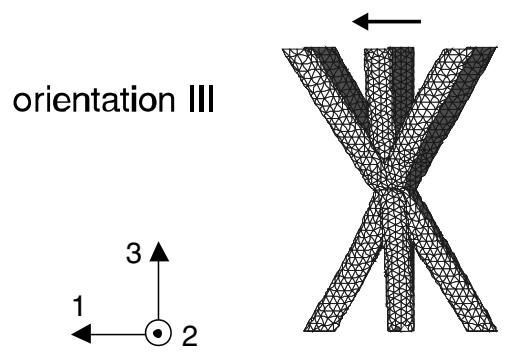

(f)

Fig. 7. The configurations subject to shear strain, $\Delta / H=0.088$, in three orientations for tetragonal and Kagomé cores. In the configuration in shear for orientation I $(a, b)$, the strain is beyond the load maximum for the tetragonal topology. Note that the tetragonal core demonstrates plastic buckling of the compressed truss. The configurations in shear for orientation II (c,d) indicating that there is no plastic buckling and that the most highly stressed trusses stretch in tension. The configurations in shear for orientation III (e,f) indicating the similarity with orientation I. 
$\widehat{F}_{\mathrm{s}} / \pi \sigma_{\mathrm{Y}} R^{2} \approx 1.1$ for the $\mathrm{Cu} / \mathrm{Be}$ alloy. In these orientations, the most highly stressed trusses are in compression, and the load peak is associated with plastic buckling (Fig. 7). Orientation II exhibits hardening, similar to that for the Kagomé core, attaining the same loads (Fig. 6a). In this orientation, the most highly stressed trusses are in tension. These trusses stretch and strain harden, allowing the load to increase with an increase in the imposed displacement.

The unload/reload simulations exhibit the same stress/strain envelope as in the monotonic curve (Fig. 8). The unloading modulus for the tetragonal core decreases in the post-buckling regime, while that for the Kagomé core remains the same throughout.

\section{Conclusions}

Numerical simulations have been performed of the mechanical responses of two truss structures (tetragonal and Kagomé) subject to compression and shear. Responses of both structures are initially isotropic, but only the Kagomé core maintains the isotropy after yielding; it strain hardens and is resistant to plastic buckling in compression and shear. The tetragonal core is anisotropic in shear with two soft orientations, both governed by the onset of plastic buckling. Accordingly, the Kagomé structure has the greater load capacity and appears to be a superior core structure for ultra-light panels. The basic predictions of the model are confirmed in a companion article (Wang et al., 2003).

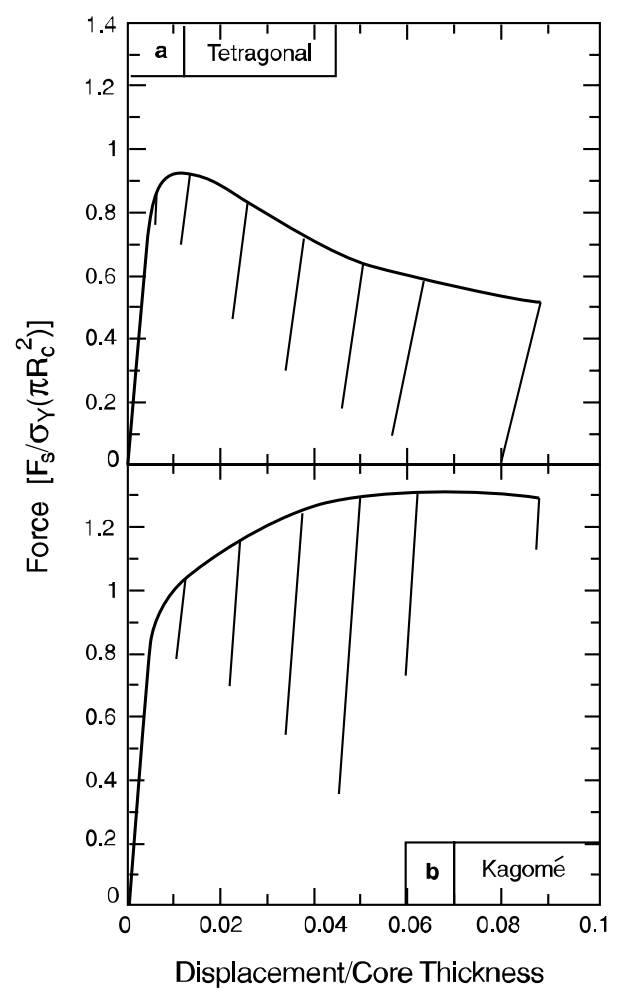

Fig. 8. Unload/reload simulations for the tetragonal core (a) and Kagomé core (b) in shear orientation I. 


\section{References}

Ashby, M.F., Evans, A.G., Fleck, N.A., Gibson, L.J., Hutchinson, J.W., Wadley, H.G.N., 2000. Metal Foams: A Design Guide. Butterworth-Heinemann, Boston.

Chiras, S., Mumm, D.R., Evans, A.G., Wicks, N., Hutchinson, J.W., Dharmasena, K., Wadley, H.G.N., Fichter, S., 2002. The structural performance of optimized truss core panels. Int. J. Solids Struct. 39, 4093-4115.

Deshpande, V.S., Fleck, N.A., 2001. Collapse of truss core sandwich beams in 3-point bending. Int. J. Solids Struct. 38, 6275-6305.

Deshpande, V., Fleck, N.A., Ashby, M.F., 2001. Effective properties of the octet-truss lattice material. J. Mech. Phys. Solids 49, 17471769.

Evans, A.G., 2001. Mater. Res. Bull. 26, 790-797.

Evans, A.G., Hutchinson, J.W., Ashby, M.F., 1998. Multifunctionality of cellular metal systems. Progr. Mater. Sci. 43, 171-221.

Evans, A.G., Hutchinson, J.W., Fleck, N.A., Ashby, M.F., Wadley, H.G.N., 2001. The topological design of multifunctional cellular metals. Progr. Mater. Sci. 46, 311-327.

Hashin, Z., 1965. J. Mech. Phys. Solids 13, 119.

Hashin, Z., Shtrikman, S., 1963. J. Mech. Phys. Solids 11, 127.

Hyun, S., Torquato, S., 2002. Optimal and manufacturable two-dimensional, Kagome-like cellular solids. J. Mater. Res. $17,137$.

Wallach, J.C., Gibson, L.G., 2001. Mechanical behavior of a three-dimensional truss material. Int. J. Solids Struct. 38, 7181-7196.

Wang, J., Evans, A.G., Dharmasena, K., Wadley, H.N.G., 2003. On the performance of truss panels with Kagomé cores. Int. J. Solids Struct., in press.

Wicks, N., Hutchinson, J.W., 2000. Optimal truss plates. Int. J. Solids Struct. 38, 5165-5183. 